\title{
A STEEL-SCINTILLATOR COUNTER TO DETECT NEUTRONS*
}

\author{
M. L. MARSHAK ${ }^{\dagger}$ and P. SCHMÜSER ${ }^{+}$ \\ Harrison M. Randall Laboratory of Physics, The University of Michigan, Ann Arbor, Michigan 48IO4, U.S.A.
}

Received 29 June 1970

A steel-scintillator sandwich counter was used as a simple and highly efficient detector for both neutrons and protons. The validity of simple approximations for the detection efficiency

\section{Introduction}

The detection of high energy neutrons by a method which is simultaneously simple and highly efficient (with known efficiency) has proven difficult. In a recent experiment at the Argonne $\mathrm{ZGS}^{1}$ ), we have measured the ratio of proton-neutron to proton-proton elastic scattering, using a proton beam and a deuterium target. The forward scattered protons were detected by a $35 \mathrm{~m}$ magnetic spectrometer; both recoil neutrons and protons were detected by a steel-scintillator sandwich counter. The sandwich counter inexpensively fulfilled the experimental requirements for an efficient and well-calibrated detector of neutrons and protons. To first approximation the efficiency for protons was 100 percent and for neutrons the efficiency was simply governed by the number of interaction lengths in the counter. Indeed, as the amount of material in the counter increased, knowing the precise number of interaction lengths became less important.

In other experiments the best calibration of detection efficiency has been attained with large-volume scintillation counters, both liquid and solid ${ }^{2,3}$ ). Yet these counters had a low total detection probability and the large amount of scintillator required was expensive. When precise resolution of the neutron trajectory was necessary, thick plate optical spark chambers were used $^{4-6}$ ), but these chambers sacrificed simplicity and the possibility of "on-line" analysis. Thick plate wire chambers could not be easily utilized since the many tracks from an inelastic interaction in the plates tended to swamp both the digital readout circuitry and the computer track reconstruction programs. For ultra-high energy cosmic rays ${ }^{7-8}$ ), total absorption ionization calorimeters, which depend on the develop-

* Work supported by the U.S. Atomic Energy Commission.

+ Present address: School of Physics, University of Minnesota, Minneapolis, Minn. 55455, U.S.A.

+ Present address: Department of Physics, University of Hamburg, 2000 Hamburg-50, W. Germany. was investigated by experimental tests and a detailed Monte Carlo calculation of the nuclear and electromagnetic cascades in the counter.

ment of an electromagnetic cascade, could be used both to detect neutrons and to provide crude energy resolution. For incident neutrons with momenta of only a few $\mathrm{GeV} / c$, total absorption counters have proven unsuitable because the electromagnetic cascade does not dominate the energy deposition process.

The steel-scintillator sandwich counter, used in this experiment, was based primarily on the nucleon-meson cascade, rather than the electromagnetic shower used by the calorimeter. Previous experiments with sandwich counters $^{9,10}$ ) have utilized first-order, intuitive ideas about the detection efficiency. To test the applicability of these concepts, we used a series of measurements to validate a Monte Carlo computer simulation of the sandwich counter. The computer program then calculated the detailed efficiency functions required in the experiment.

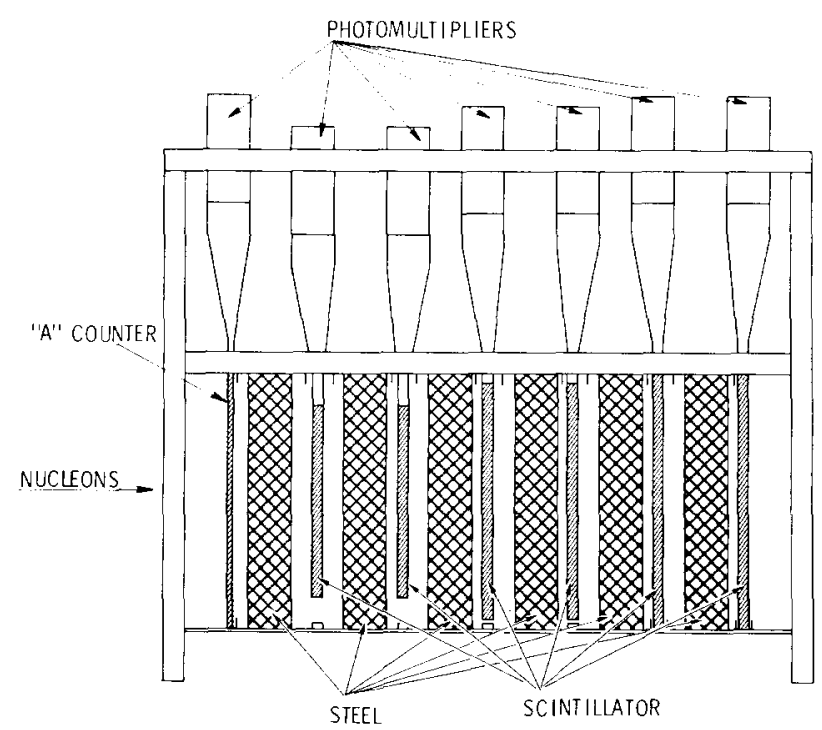

Fig. 1. A side view of the steel-scintillator sandwich counter with the " 12 in. total -2 in. between scintillators" steel configuration. 


\section{The sandwich counter}

The physical configuration of the counter in a side view is shown in fig. 1. Six one-half inch Pilot $Y$ scintillators were interspersed among sets of steel plates $15^{\prime \prime}$ wide by $12^{\prime \prime}$ high. The first two scintillators were $12^{\prime \prime}$ by $9^{\prime \prime}$, the next two were $14^{\prime \prime}$ by $11^{\prime \prime}$ and the back two were $15^{\prime \prime}$ by $12^{\prime \prime}$. The nominal center-to-center separation between scintillators was $4 "$. The thickness of steel between any two scintillators was adjustable from $0^{\prime \prime}$ to $3^{\prime \prime}$ in $\frac{1}{4}^{\prime \prime}$ increments.

Attached to the front of the sandwich counter was the A counter, a $15^{\prime \prime} \times 12^{\prime \prime} \times \frac{1^{\prime \prime}}{4}$ scintillator, which determined the charge of the incident nucleon. Placing the A counter so close to the steel of the sandwich counter confused the identification of protons and neutrons. A neutron could interact in the steel, backscatter charged secondaries into the A counter and forward scatter secondaries into the sandwich counter, thus faking the signature of a proton. Moving the A counter a few feet from the steel of the sandwich counter would eliminate this ambiguity, because the timing would no longer be identical for neutrons with backscattering and true protons incident on the counter.

The six scintillators of the sandwich counter were each optically connected through a constant-area lucite light pipe, which minimized losses, to an RCA 7746 photomultiplier. The A counter used a similar light pipe attached to an Amperex 56UVP phototube. All photomultiplier bases shared a high current supply for the last dynode and used Zener diodes on the next-to-last dynode to eliminate voltage sag at counting rates up to $1 \mathrm{MHz}$.

The photomultiplier voltages were set to equalize the output pulse heights for single minimum-ionizing particles. With all steel removed from the counter, high voltage plateau curves were run on one counter in coincidence with two others or with the spectrometer. A spectrometer coincidence indicated that a minimum-ionizing recoil proton from a pp elastic scattering passed through the counter. This process was iterated with various counter combinations until a consistent set of voltage settings emerged. Another method used a linear gate and a pulse height analyzer to directly equalize the output pulse heights from the six phototubes. The opening of the gate was triggered by a spectrometer coincidence. The settings from these two methods were essentially identical.

When used to detect neutrons, the outputs from the six photomultipliers were each fed into separate Chronetics $100 \mathrm{MHz}$ discriminators. The discriminator outputs were folded together by two-stage logical OR circuitry, which provided a sandwich counter output if a minimum-ionizing particle passed through any one of the scintillators.

In operation the sandwich counter was placed $156^{\prime \prime}$ from a $3^{\prime \prime}$ liquid deuterium target in the new second extracted proton beam of the ZGS. The counter axis made an angle of $49^{\circ}$ to the beam. The counter was separated from the proton tunnel by an $18^{\prime \prime}$ steel wall with a hole cut to allow the nucleons to reach it. For $10^{10}$ protons per pulse, the $\mathrm{A}$ counter singles rate and the sandwich counter OR counting rate were both of order $5 \times 10^{5}$ during the $550 \mathrm{msec}$ spill.

\section{The Monte Carlo simulation}

The detailed calibration of the sandwich counter was accomplished by a Monte Carlo simulation of the nuclear cascade; the validity of the calculation was tested by experimentally determining the proton and neutron counting rates for seven steel configurations. Since the counter depended on inelastic interactions it was not perfectly efficient nor was its efficiency constant over its entire active area. The detection efficiency profile of the counter was also, in general, dependent on the momentum of the incident nucleon.

The nuclear cascade model was based on previous calculations ${ }^{11,12}$ ) made to determine adequate accelerator shielding thicknesses. The incident particle and all subsequently produced particles were followed until their momentum was less than $10 \mathrm{MeV} / \mathrm{c}$, although the model was inaccurate at such low momenta since nuclear reactions were not included. The major mechanism considered was inelastic scattering. The parameters which govern these interactions in steel, including the cross-section and the momentum and angular distributions of the produced particles, were identical to those used by $\operatorname{Ranft}^{11}$ ). For inelastic scattering in air and scintillator, the interaction length used was the one published in standard tables ${ }^{13}$ ). The momentum and angular distributions for steel interactions were also used for scattering in air and scintillator. Because most of the inelastic scattering took place in the steel, this simplification introduced a negligible error. The distribution of types of particles produced was set by multiplicities taken from experimental data in the $1 \mathrm{GeV} / c$ region ${ }^{14}$ ). The assumption that neutron production from a complex nucleus equaled proton production and that $\pi^{\circ}$ production equaled $\pi^{+}$production was used to determine unobserved multiplicities. The total number of particles 


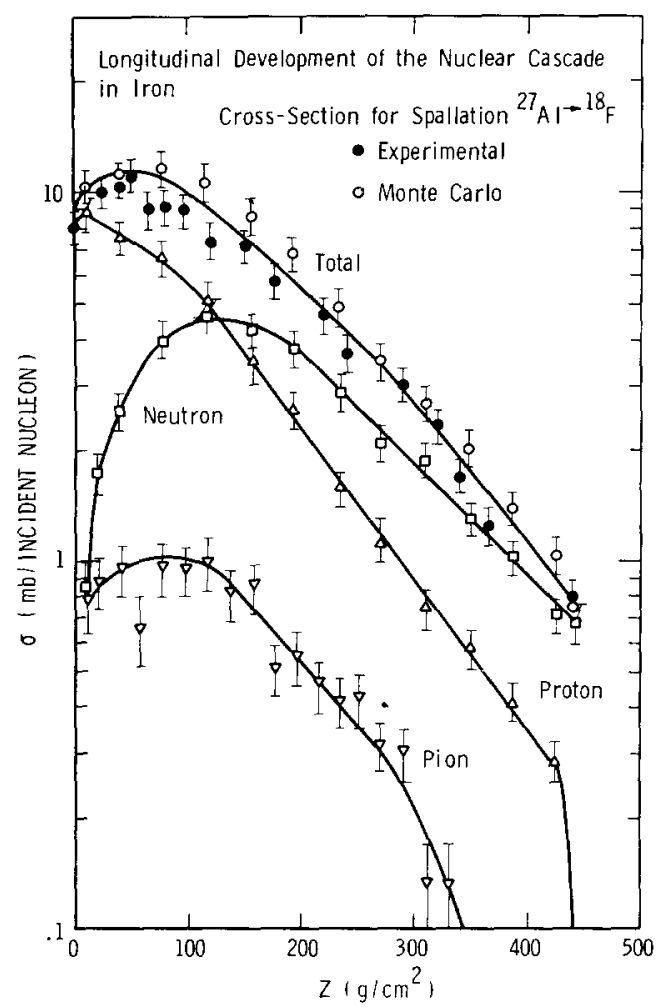

Fig. 2. The longitudinal development of the nuclear cascade from $1 \mathrm{GeV}$ protons in a steel block. Errors shown are statistical only.

produced in each interaction was governed by the requirement that the kinetic energy be conserved.

The results of the Monte Carlo simulation were generally insensitive to small changes in the inelastic scattering parameters. The only parameter with a significant influence on the final result was the inelastic cross-section which affected the efficiency in the expected exponential manner. The results of the model were insensitive to raising the low momentum cut-off from $10 \mathrm{MeV} / c$ to $50 \mathrm{MeV} / c$, to changing all the momentum and angular spectra parameters by 25 percent and to varying the relative multiplicities of nucleons and pions by 25 percent.

The possibility of elastic scattering for all particles was also considered using the cross-sections given in ref. 11 . The angular scattering was computed according to the distribution $\left.{ }^{15}\right) \exp \left(-10 \beta_{\text {c.m. }}^{2} A^{\frac{2}{3}} p_{1}^{2}\right)$. The recoil particle was ignored which resulted in a negligible error in the case of true elastic scattering due to the massiveness of the nucleus. Only a small error resulted for quasi-elastic scattering because most such interactions involve a small momentum transfer.

The effect of multiple Coulomb scattering was calculated according to a Gaussian distribution. Ionization losses were also calculated for charged particles using a three-term empirical fit to published stopping-power tables ${ }^{16}$ ). The loss calculated for each particle was determined by its velocity and momentum.

The decay of charged pions was not considered, since the small size of the counter made this process relevant for only low energy pions. These particles had a very short range in steel, as did the low energy muons which would result from a decay. The error from neglecting this process was therefore small. The decay of neutral pions into two gammas was calculated and the electromagnetic shower resultant from the gammas was considered. The shower was approximated by a one dimensional model, with the assumption of normal statistics. The mean and variance of the probability of finding a certain number of electrons at a certain depth in radiation lengths were interpolated

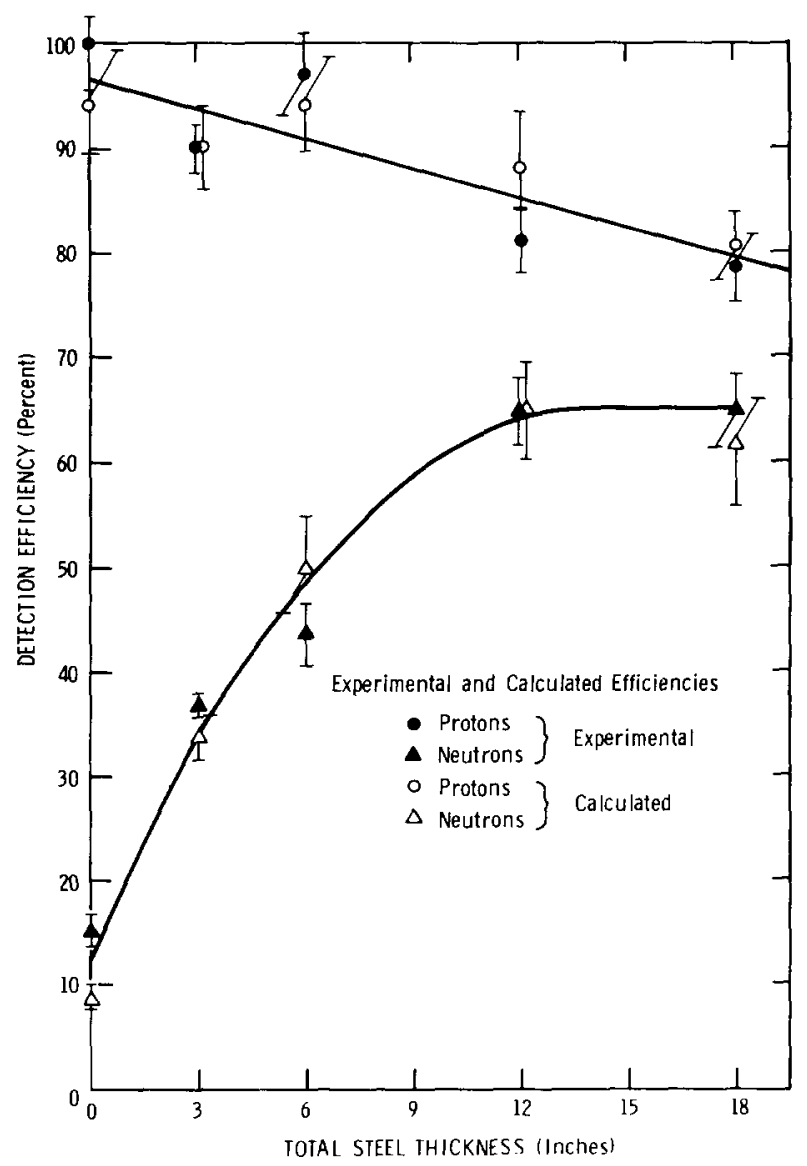

Fig. 3. Comparison of the experimental and calculated counting rates for the sandwich counter with different steel configurations. Recoil nucleon and backscattering effects are included in the calculated rates. 
from the results of Monte Carlo calculations for copper ${ }^{17}$ ). This treatment of the electromagnetic cascade was simplified but the effect on the final results was small since only about five percent of the neutrons were detected solely by an electromagnetic shower.

The collective reliability of the assumptions about the nuclear cascade was tested by using the Monte Carlo model to simulate an experiment without adjusting any of the model parameters ${ }^{18}$ ). The program calculated the ${ }^{18} \mathrm{~F}$ activity induced by $1 \mathrm{GeV}$ protons in thin aluminum foils placed within a block of steel $1 \mathrm{ft}$ by $1 \mathrm{ft}$ in cross-section. This calculation used detailed single particle spallation cross-sections as a function of kinetic energy, which have been measured for various particles incident on the aluminum ${ }^{12}$ ). The comparison with experiment as a function of longitudinal depth is shown in fig. 2 .

\section{Results}

To test the validity of the Monte Carlo model, the overall detection efficiency of the sandwich counter was calculated as a function of the steel configuration. This computation involved several factors - the detection efficiency at the center of the counter, the drop in efficiency near the edges, the distribution of incoming nucleons and the possibility of a backscattering from an incident neutron into the A counter. For simplicity the incoming neutrons were assumed to be uniformly distributed over the counter area with random directions consistent with their coming from a target. To compare with calculated efficiencies, the experimental data were corrected for accidental coincidences; the effect of faked proton triggers due to backscattering into the A counter was also considered. The comparison of calculated and experimental efficiencies as a function of the total amount of steel in the counter is shown in fig. 3. The normalization between the experimental proton and neutron efficiencies assumed that the ratio of incoming protons to neutrons was $0.95^{1}$ ). The lack of agreement at the no-steel point for neutrons was not unexpected. A model based on the nuclear cascade in steel should not be able to predict experimental efficiencies with no steel present. The comparison between calculated and experimental efficiencies for a fixed amount of steel distributed in different amounts between scintillators showed similar agreement.

The Monte Carlo simulation was then used to calculate detailed detection efficiency profiles for the " 12 in. total -2 in. between scintillators" steel configuration, which was the arrangement used in the pn scattering experiment. The program was also used to calculate a correction for the misidentification of protons and neutrons resultant from backscattering into the A counter. The high singles rate observed in the sandwich counter motivated the consideration of triggering schemes other than one particle in any one scintillator. Detection efficiencies were also calculated for the trigger requirements "any one counter", "any two counters", "any two adjacent counters", "any three counters" and "at least two ionizing particles detected". Any of these schemes would result in a much lower sandwich counter singles rate. Fig. 4 shows the calculated detection profile for $1.8 \mathrm{GeV} / \mathrm{c}$ neutrons incident on the horizontal axis of the counter

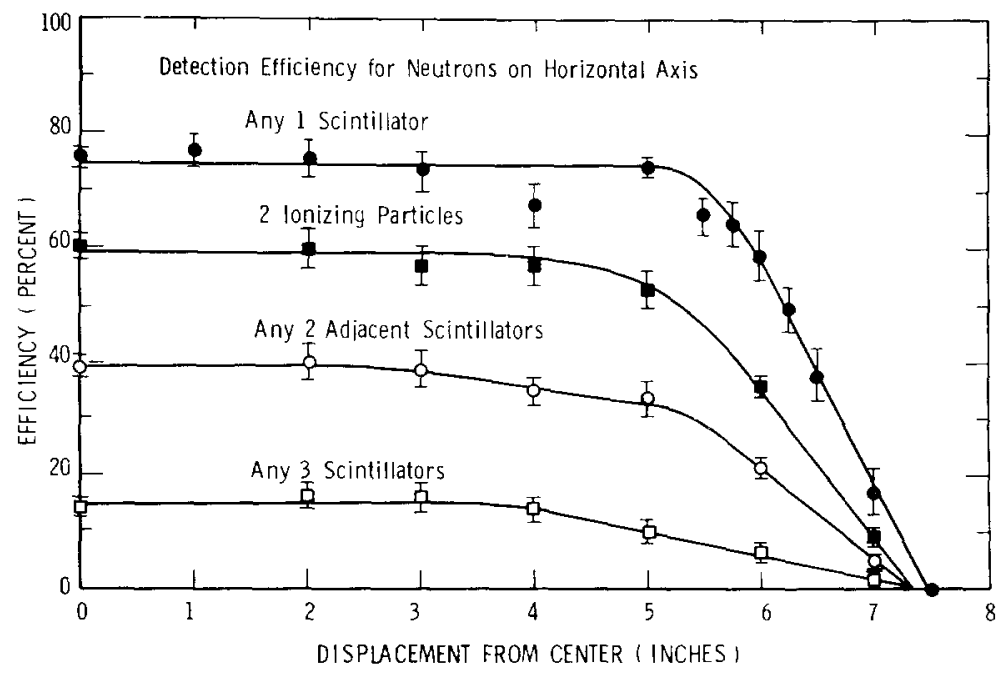

Fig. 4. Detection efficiency for neutrons along the horizontal axis of the sandwich counter for various trigger requirements. 
as a function of displacement from the center for the various detection schemes. The detection efficiency offaxis was well-represented by the equation

Eff. $(x, y)=$

Eff. $(x, y=0) \times$ Eff. $(x=0, y) /$ Eff. $(x=0, y=0)$.

The central detection efficiency as a function of incident neutron momentum for the same " $12 \mathrm{in} .-2$ in." steel arrangement but varying trigger requirements is shown in fig. 5. The "any one counter" triggering scheme used in the pn experiment had the advantage of a uniform sandwich counter response over a large central area and over the incident momentum range from 1.8 to $5 \mathrm{GeV} / c$. The non-central detection efficiency profile was also invariant for this triggering scheme over the same momentum range.

To a first approximation then, the detection efficiency for neutrons for the "any one counter" triggering scheme over a large range of incident momenta depended only on two parameters - the total amount of steel in the counter and the amount of steel between any two scintillators. The inelastic interaction length, which was measured in other experiments as $6.4^{\prime \prime 11}$ ), characterized the probability that a neutron would interact at all in the counter. The other characteristic length was an absorption length which measured the probability that the charged secondaries from a neutronsteel interaction would be absorbed before they could reach a scintillator. From the results of the Monte Carlo calculation, confirmed by experiment, this

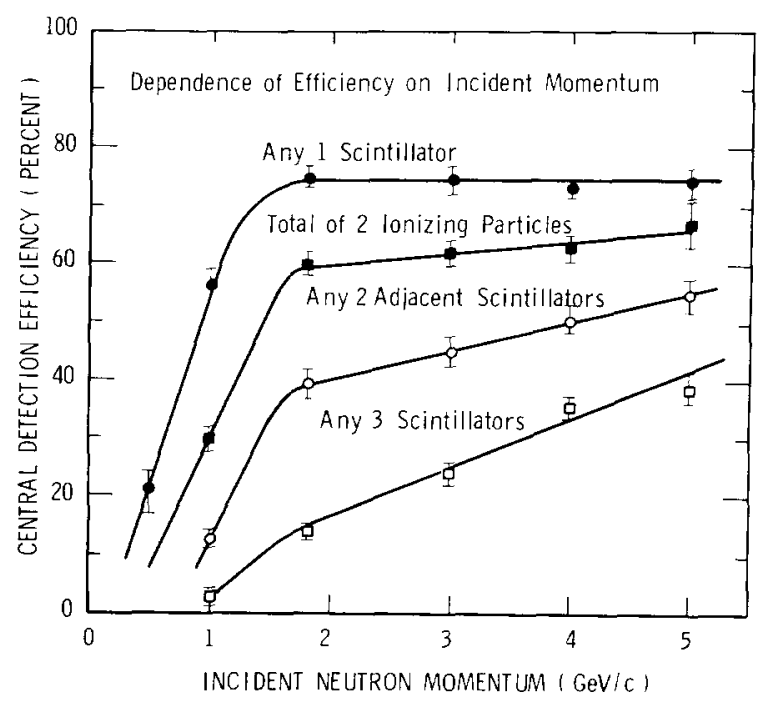

Fig. 5. Sandwich counter central detection efficiency for neutrons as a function of incident neutron momentum for various trigger requirements. length was $23^{\prime \prime} \pm 2^{\prime \prime}$. Thus the detection efficiency over a large part of the counters's sensitive area was approximately

$$
\text { Eff. }=(0.95)\left(1-\mathrm{e}^{-x / 6.4}\right)\left(\mathrm{e}^{-y / 23}\right),
$$

where $x$ is the total length of steel (inch) and $y$ is the amount of steel between any two scintillators. The coefficient 0.95 reflects the probability that a neutron might have interacted to produce only neutrals which were never detected.

The assessment of errors in the Monte Carlo calculation followed from a detailed analysis of the various reasons for counter inefficiency. For the " 12 in. -2 in." steel arrangement the computed central detection efficiency was 75 percent, with a two percent statistical error. There were two contributions to the 25 percent loss. Approximately 15 percent of the incident neutrons passed through the counter without interacting; this number depended only on the inelastic cross-section. Knowledge of that cross-section to 10 percent implied only a three percent error in the detection efficiency. This left 10 percent of the incident neutrons which interacted but were not detected because of production of neutrals, absorption of charged secondaries or scattering of secondaries outside the sensitive counter volume. Assuming a somewhat arbitrary 50 percent error in the calculation of this process implied a five percent error in the overall efficiency. Considering all these errors in quadrature resulted in a central detection efficiency for this steel configuration of $75 \pm 6$ percent.

Determination of the overall sandwich counter response for both types of nucleons involved additional errors. Calculation of "edge effects" and backscattering each introduced an independent four percent error in the calculated counting rates. For comparison with the experimental rates shown in fig. 3, and additional four percent error for accidentals correction must be added. Using these estimated errors, the comparisons shown in fig. 3 yield a chi-squared confidence level in excess of 40 percent for both protons and neutrons, with the exclusion of the no steel point for neutrons.

\section{Conclusions}

The results of the Monte Carlo calculations corrobate the general validity of the intuitive ideas about the detection efficiency of the sandwich counter. The relatively long length $-23^{\prime \prime}$ - for absorption of secondaries indicates that this is not a serious effect for any reasonable amount of steel between scintillators. The five percent loss in efficiency due to formation of 
neutrals is relatively independent of the amount of steel. The Monte Carlo calculation also indicates that for the triggering scheme used the detection efficiency for neutrons is relatively constant up to one inch from the edge of the scintillator. The sandwich counter has the considerable advantage that although a judicious mixture of steel and scintillator will optimize its performance for any given momentum particle, to first approximation a straightforward approach is all that is necessary.

We acknowledge the assistance of J. L. Day, P. Kalbaci, A. D. Krisch, G. J. Marmer, J. K. Randolph and L. G. Ratner in performing the measurements described here. We also thank Drs. Krisch and Randolph for reading this manuscript and for their helpful comments. We deeply appreciate the assistance of the ZGS staff.

\section{References}

1) J. L. Day, P. Kalbaci, A. D. Krisch, M. L. Marshak, P. Schmüser, J. K. Randolph, G. J. Marmer and L. G. Ratner, Phys. Rev. D (Nov. 1970) to be published.

2) R. Hofstadter, Stanford HEPL Report 599 (March 1969).

3) A. S. L. Parsons, P. Trueol, P. A. Berardo, R. P. Haddock, L. Verhey and M. E. Zeller, UCRL-19259 (July 1969).

4) R. C. Chase, E. Coleman, H. W. J. Courant, E. Marquit, E. W. Petraske, H. Romer and K. Ruddick, Phys. Rev. Letters 22 (1969) 1137.
5) M. L. Perl, J. Cox, M. J. Longo and M. N. Kreisler, Phys. Rev. D 1 (1970) 1857.

6) B. G. Gibbard, L. W. Jones, M. J. Longo, J. R. O'Fallon, J. Cox, M. L. Perl, W. T. Toner and M. N. Kreisler, Phys. Rev. Letters 24 (1970) 22.

7) V.S. Murzin, in Progress in elementary particle and cosmic ray physics $I X$ (eds. J. G. Wilson and S. A. Wouthuysen; North-Holland Publ. Co., Amsterdam, 1967).

8) D. E. Lyon and A. Subramanion, University of Michigan Preprint (1967).

9) Yu. V. Galaktionov, L. G. Landsberg and V. A. Lyubimov, Nucl. Instr. and Meth. 39 (1966) 351.

10) L. W. Jones, Tech. Report 32 (University of Michigan, 1968).

11) J. Ranft, Nucl. Instr. and Meth. 48 (1967) 133.

12) T. W. Armstrong and R. H. Alsmiller, Jr., Nucl. Sci. Eng. 33 (1968) 291.

13) N. Barash-Schmidt, A. Barbaro-Galtieri, L. R. Price, M. Roos, A. H. Rosenfeld, P. Soding and C. G. Wohl, UCRL8030 (Jan. 1969).

14) V.S. Barashenkov, V. M. Maltsev, I. Patera and V. D. Toneev, Fortschr. Physik 14 (1966) 357.

15) A. D. Krisch, Phys. Rev. Letters 19 (1967) 1149.

16) W. H. Barkas and M. J. Berger, NASA SP-3013 (1964). The empirical fit used was

$$
\begin{aligned}
& \frac{\mathrm{d} E}{\mathrm{~d} x}=\left(\frac{\mathrm{d} E}{\mathrm{~d} x}\right)_{\min }\left[54.1 \mathrm{e}^{-9.46 \sqrt{ } \beta p}+6.82 \mathrm{e}^{-2.13 \sqrt{ } \beta p}\right] \\
& \text { or } \frac{\mathrm{d} E}{\mathrm{~d} x}=\left(\frac{\mathrm{d} E}{\mathrm{~d} x}\right)_{\min }, \text { whichever is greater. }
\end{aligned}
$$

17) D. F. Crawford and H. Messel, Nucl. Phys. 61 (1965) 145. 18) S. P. Shen, BNL-8721 (1964). 